\title{
Brecha en el uso de tecnologías de la información y comunicación (TIC) básicas y modernas entre estudiantes y docentes en universidades ecuatorianas
}

\author{
Paredes-Parada, Wladimir \\ Brecha en el uso de tecnologías de la información y comunicación (TIC) básicas y modernas entre estudiantes y \\ docentes en universidades ecuatorianas \\ Revista Educación, vol. 43, núm. 1, 2019 \\ Universidad de Costa Rica, Costa Rica \\ Disponible en: http://www.redalyc.org/articulo.oa?id=44057415009 \\ DOI: https://doi.org/10.15517/revedu.v43i1.27423
}

Esta obra está bajo una Licencia Creative Commons Atribución-NoComercial-SinDerivar 3.0 Internacional. 


\title{
Brecha en el uso de tecnologías de la información y comunicación (TIC) básicas y modernas entre estudiantes y docentes en universidades ecuatorianas
}

\author{
Gap in the use of basic and modern information and communication technologies (ICT) among students and \\ teachers in Ecuadorian universities
}

Wladimir Paredes-Parada
Universidad Tecnológica Israel,,Ecuador
wparedes@uisrael.edu.ec
iD http://orcid.org/0000-0003-1441-4426

DOI: https://doi.org/10.15517/revedu.v43i1.27423

Redalyc: http://www.redalyc.org/articulo.oa?id=44057415009

Recepción: 13 Diciembre 2016

Aprobación: 25 Noviembre 2018

\section{RESUMEN:}

El presente trabajo permite ver la brecha que existe entre los estudiantes y docentes, en cuanto al uso y manejo de las tecnologías de la información y comunicación en el proceso de enseñanza aprendizaje. Se realizó una investigación cuantitativa de tipo descriptivointerpretativo y analítico ex-post-facto y se presentan datos obtenidos mediante la aplicación de un cuestionario; basado en investigaciones previas las cuales abarcan las áreas de manejo de equipos, software de escritorio e internet especializado en educación e investigación; se lo aplicó a 133 docentes y 906 estudiantes de dos universidades del Ecuador, una de Quito y otra de Guayaquil. Se pudo observar que el alumnado usa mayormente la tecnología basada en el Internet, sin que esto signifique que lo hacen con fines académicos (muchas veces para recreación); sin embargo, los docentes aprovechan o le dan mayor utilidad a las herramientas tradicionales en las actividades académicas como las PC, proyectores y demás. Podemos concluir que en el uso de la tecnología básica la brecha es pequeña en estos dos grupos, pues los docentes están en constante capacitación en tecnología, principalmente porque las IES buscan la mejora continua en su calidad educativa, además existen políticas y leyes del Estado Ecuatoriano que permiten la evaluación constante de las Universidades en buscan de la calidad académica y mejora del proceso de generación del conocimiento para la obtención de una acreditación Universitaria y en varios de los indicadores de esta evaluación apunta al uso de la tecnología. Se recomienda canalizar el apropiado uso de las tecnologías de vanguardia que realizan los estudiantes para que sea con fines académicos y no de ocio; partiendo de correctas políticas tecnológicas que deben incorporar los docentes en el proceso de enseñanza aprendizaje y esto solo se puede realizar una vez reducida la brecha tecnológica entre docentes y estudiantes.

Palabras Clave: TIC, brecha tecnológica, enseñanza aprendizaje, universidades.

\section{Abstract:}

The article shows the gap between students and teachers regarding the use and management of basic and advanced information and communication technologies in the teaching-learning process. A quantitative investigation descriptive-interpretative and analytical type was carried ex-post-facto. Data obtained through the application of a questionnaire has been presented, based on previous research that included the areas of equipment management, desktop software and internet specialized in education and research. The questionnaire was applied to 133 teachers and 906 students from two universities in Ecuador, one from Quito and one from Guayaquil. It was observed that students use mostly Internet-based technology, but this does not mean that they do it for academic purposes (often for recreation). However, teachers take advantage or give more utility to traditional tools in academic activities such as PCs, projectors and so on. In the use of basic technology, the gap is small in these two groups. Teachers are on constant training on technology, mainly because the study centers look for continuous improvement in their educational quality. In addition, there are policies and laws of the Ecuadorian State that allow the constant evaluation of universities that look for the academic quality and improvement in the process of generating knowledge to obtain a University accreditation. In several indicators of this evaluation are the use of basic communication technology. It is recommended to direct the appropiate use of new technologies that students make for academic purpose not for leisure, taking into consideration the correct technological policies that teachers must include in the teaching learning process. This can be done if the gap between teachers and students would be reduced.

KEYWORDS: ICT, technological gap, teaching-learning, universities. 


\section{INTRODUCCIÓN}

La presente investigación tiene como propósito generar una conciencia en las autoridades de Educación Superior, así como en las IES y docentes de la importancia que tiene el reducir la brecha tecnológica entre estudiantes y docentes, pues así se podrá potenciar y mejorar el proceso de enseñanza aprendizaje de los estudiantes, para que este genere valor de desarrollo local, así como la masificación del conocimiento a las poblaciones menos favorecidas.

Las universidades deben orientar todos sus objetivos hacia la sociedad del conocimiento (UNESCO, 2005) porque la comunicación científica y la enseñanza depende fundamentalmente de las tecnologías de la información y comunicación (TIC). Esta afirmación identifica claramente las necesidades actuales que surgen a partir de las TIC y debería ser una guía para quienes son parte de la Educación Superior en el mundo.

Desde el año 2008, en el Ecuador se han realizado cambios a nivel gubernamental en función del mejoramiento de la calidad en las Instituciones de Educación Superior (IES) por parte del gobierno; en su informe del 24 de abril del 2013, establece tres niveles de universidades por la oferta académica y las agrupa en universidades: de grado; de grado y posgrado; y, de posgrado; en cada uno de estos niveles se categorizan a las IES por $\mathrm{A}, \mathrm{B}, \mathrm{C}$ o D , según la calidad académica.

Para efectos de esta investigación, se han tomado datos en dos universidades de las principales ciudades del Ecuador: Quito (Universidad Tecnológica Israel) y Guayaquil (Universidad Laica Vicente Rocafuerte); ambas universidades se ubican en la categoría $\mathrm{C}$ en el grupo de oferta académica de grado y posgrado. A continuación, un breve detalle de las características de cada una de ellas.

Las dos universidades tienen sistemas de Educación Virtual en plataforma Moodle y E-ducativa; donde los docentes utilizan estas herramientas como apoyo para impartir sus clases. También se observa un acceso permanente a Internet que cuenta con un ancho de banda técnicamente aceptable, una distribución de Wifi bien administrado, bibliotecas apropiadas e infraestructura pedagógica que aporta a un entorno de calidad.

En este contexto se inscribe la investigación que permite determinar la brecha tecnológica en el uso de TIC en estudiantes y docentes en las universidades; esta es de suma importancia y deben ser el punto de partida para las políticas institucionales, a fin de que se pueda realizar programas y proyectos idóneos que permitan reducir la brecha tecnológica entre estudiantes y docentes, de manera que se considera relevante el objetivo de mejorar los procesos de enseñanza aprendizaje, debido a que el uso de herramientas tecnológicas en el aula mejora la motivación en los estudiantes. Además, el uso de las herramientas tecnológicas en el aula, por parte de los docentes en el proceso de enseñanza aprendizaje, es un recurso considerado en el modelo de evaluación del Consejo de Evaluación, Acreditación y Aseguramiento de la Calidad de la Educación Superior (CEAACES), en los indicadores correspondientes a los criterios de Docencia y Recursos tecnológicos.

\section{ANTECEDENTES}

Se incluyen experiencias internacionales relacionadas con el uso intensivo de las TIC en diversas instituciones de educación de diferentes niveles (con énfasis en la Educación Superior) y se ordenan cronológicamente; porque "La experiencia acumulada y el seguimiento de algunas prácticas exitosas en el uso educativo de las TIC nos indican algunos de los factores clave que pueden estar implicados en una integración adecuada de estas tecnologías en la formación universitaria" (Sigalés, 2004, p. 1).

Morales, Trujillo y Raso (2015), analizan el impacto del uso de las TIC, a partir del Espacio Europeo de Educación Superior y tratan de conocer la valoración del alumnado de la Facultad de Ciencias de la Educación de la Universidad de Granada, sobre la importancia de las TIC en los procesos de enseñanza aprendizaje y, conocer las razones del profesorado de la Facultad de Ciencias de la educación de la Universidad de Granada, para integrar las TIC en el proceso de enseñanza-aprendizaje. 
Fu (2013), desde la perspectiva teórica, presenta una visión crítica y, el estado del arte de la investigación en TIC, hace referencia a los estudios de críticos sobre los méritos de la integración de las TIC en las escuelas, los obstáculos o retos enfrentados en el uso de las TIC. Se determinan los factores que influyen en la integración exitosa de las TIC, los beneficios y las barreras presentadas y además muestra las actitudes de los profesores, las percepciones, y la confianza en el uso de las TIC.

Rozo y Prada (2013), describen la estrategia de incorporación de TIC a la formación inicial docente por vía de reformas educativas que intentan una ampliación de cobertura y mejoramiento de la calidad en instituciones de Educación Superior e institutos de formación pedagógica en los países de la Región Andina: Bolivia, Colombia, Ecuador, Perú y Venezuela; presenta 23 experiencias, en cuatro de los cinco países mencionados, que formaron parte del trabajo Formación docente y TIC, una aproximación al mapeo en la Región Andina.

Fombona y Pascual (2011) presentan una investigación de carácter cualitativo que analiza y describe la introducción de las TIC en la Universidad Nacional Autónoma de México (UNAM). Para realizar el estudio se diseñó y aplicó un instrumento tipo encuesta con preguntas abiertas a 192 profesores de Escuelas y Facultades de la Universidad Autónoma de México elegidas al azar.

Lareki, Martínez de Morentin y Amenabar (2010), pretenden resaltar la importancia que pueden adquirir la integración de las TIC de parte de los docentes en las aulas universitarias. Propone 10 plataformas online, gratuitas y de fácil manejo, para impulsar la información entre los alumnos y los docentes bajo una metodología en línea; este nuevo modelo pedagógico se apoyaría en el concepto entendido como blendedlearning.

Kalogiannakis (2010) presenta desafíos de los docentes en el uso de TIC en las universidades y los cambios surgidos en la enseñanza a partir de un estudio exploratorio realizado en el marco de un programa nacional desarrollado en Grecia, se aplicó un cuestionario estructurado; se plantea la necesidad de cambio de políticas educativas.

Van Der Vyver (2009) realiza un estudio centrado en el uso de TIC desde el factor estudiante que como nuevo profesional debe ser un solucionador de problemas que comprende los negocios y la tecnología; en particular, se muestra cómo la tecnología puede resolver problemas de negocios; además, señala que el perfil del estudiante de las TIC debe ser adaptable y posibilite respuestas variadas, autonomía y actúa como un agente de cambio en la organización.

Nordkvelle y Olson (2005) presentan la visión ética en el uso de las TIC en la enseñanza universitaria; señalan que se atribuye el fracaso al factor docente por la resistencia o falta de voluntad como factores preponderantes, aunque, existe una necesidad de análisis desde un ángulo diferente. Se plantea la necesidad de cambio de políticas educativas e incluir a la tecnología, su práctica y uso como parte de los valores morales del docente en la enseñanza.

Venables y Tan (2012) realizan un estudio en la Universidad Victoria (Australia) y muestran las dificultades surgidas a partir de estos desafíos locales, nacionales e internacionales con respecto a la las TIC y proponen una serie de programas informáticos necesarios para la actualización del docente que permitan el manejo de la escritura, cálculos numéricos e información informática autónomamente en diferentes contextos y medios.

Nordkvelle y Olson (2005) presentan la visión ética del uso de las TIC en la enseñanza universitaria; señala que se atribuye el fracaso al factor docente por la resistencia o falta de voluntad como factores preponderantes, pero existe una necesidad de un análisis desde un ángulo diferente se plantea la necesidad de cambio de políticas educativas e incluir a la tecnología, su práctica y uso como parte de los valores morales del docente en la enseñanza del alumno.

Martínez y Acosta (2011) pretenden resaltar la importancia que pueden adquirir la integración de las TIC de parte de alumnos y docentes en las aulas universitarias. Propone 10 plataformas online, gratuitas y de fácil 
manejo, para impulsar la información entre los alumnos y los docentes bajo una metodología en línea; este nuevo modelo pedagógico se apoyaría en el concepto entendido como blended-learning.

\section{EL USO DE TIC EN LA EDUCACIÓN UNIVERSITARIA}

El uso de TIC en la educación universitaria es uno de los tópicos más analizados en las investigaciones que se refieren al proceso de enseñanza aprendizaje y se han producido cambios y que nadie puede negar pues es un recurso didáctico importante (Guerra, González y García, 2010). Las modalidades de formación apoyadas en las TIC llevan a nuevas concepciones del proceso de enseñanza aprendizaje que acentúan la implicación activa del alumno y también cambia el rol del personal docente en un ambiente rico en TIC (Salinas, 2004); estas transformaciones hacen necesarios la realización de estudios (o propuestas) para la medición del impacto en la aplicación de las TIC (Ávila y Erazo, 2011).

Johnson, Becker, Estrada y Freeman (2015), señalan que las universidades de nuestro continente no son la excepción, hay un cambio en el paradigma de la enseñanza aprendizaje y por tal razón desde hace algún tiempo se vienen publicando guías y diagnósticos para la inclusión digital de los docentes y estudiantes de las universidades.

Justamente el uso de las TIC requiere de una profunda reflexión por las enormes posibilidades que pueden surgir en el ámbito de formación universitaria desde un planteamiento diferente (Cabero, 2015); por tal razón, como respuesta a estos retos se debe reaprender y repensar en cambios en los modelos de enseñanza aprendizaje (Rodríguez, 2010). La influencia de las TIC en la enseñanza aprendizaje fomenta propuesta tales como el Aprendizaje 2.0 (Esteve, 2016) y Universidad 2.0 (Kulakli y Mahony, 2014). Este cambio de paradigma en la enseñanza aprendizaje hace imprescindible de una alfabetización digital dirigida a docentes y estudiantes que privilegie la cultura digital sea informacional y tecnológica (Marín, Vásquez, Llorente y Cabero, 2012; Rangel y Peñalosa, 2013)

Es aconsejable que el proceso de enseñanza aprendizaje incluya los beneficios de las TIC y debe ser elemento fundamental de las políticas públicas que establezcan indicadores, diagnósticos, principios y medidas y modelos, entre otros (Paredes, 2013). Las TIC como política educativa enfatizan en las buenas prácticas para reforzar el trabajo colaborativo y cooperativo entre docentes y estudiantes (Gutiérrez, Yuste, Cubo y Lucero, 2011).

El uso de herramientas tecnológicas es imprescindible en el desarrollo del proceso educativo, algunos autores orientan sobre las plataformas gratuitas en Internet, especialmente redes sociales que promueven la interacción entre docentes y estudiantes universitarios (Martínez y Acosta, 2011; Regueyra, 2011) pues es claro que existen problemas latentes en la formación didáctica del docente en el uso de TIC (Cabero, 2014) y lo demuestran algunas sus experiencias en el uso TIC e indagan sobre la capacitación docente (Rosario y Vásquez, 2012) e incluso se utilizan Estándares UNESCO de Competencias en TIC para Docentes en dominio en el uso de las TIC (Vera, Torres y Martínez, 2014).

\section{Metodología Y ANÁLISIS DE DATOS}

La investigación se realizó en dos universidades, una de Quito y otra de Guayaquil. La Universidad Laica Vicente Rocafuerte (ULVR) con su sede matriz en Guayaquil, es una universidad cofinanciada, es decir, recibe el 30\% de su presupuesto por parte del Estado; fue Creada en 1966, en la actualidad cuenta con 8.572 estudiantes y 351 miembros de su planta académica que pertenecen a sus carreras de grado y programas de posgrado, funciona en tres modalidades de estudio: presencial, semipresencial y a distancia.

La Universidad Tecnológica Israel (UISRAEL) con su sede matriz en Quito D.M., es una universidad particular, que se crea mediante ley en 1999 , tiene dos campus universitarios y en la actualidad cuenta con 
1.632 estudiantes y 94 miembros de su planta académica que pertenecen a sus carreras de grado y programas de posgrado, funciona en tres modalidades de estudio, presencial, semipresencial y a distancia.

Los objetivos establecidos para la presente investigación están centrados en determinar la brecha que existe entre docentes y estudiantes en el uso de TIC en el proceso de enseñanza aprendizaje y si esta brecha es reducida por los impactos de las acreditaciones institucionales realizadas por parte del gobierno ecuatoriano.

Los objetivos específicos se dieron al estudiar los siguientes campos:

- Determinar el uso intensivo de TIC

- Identificar herramientas para la educación virtual

- Analizar el uso de herramientas de Internet

- Identificar las herramientas tecnológicas que mayor uso tienen en la actualidad

- Determinar las herramientas para la búsqueda y uso de la información

En las Facultades de Administración de las dos universidades del Ecuador.

Se realizó y aplicó un cuestionario a 133 docentes y 906 estudiantes en las dos universidades mencionadas. Tipo de estudio y/o Diseño del estudio.

La investigación es cuantitativa y el tipo de investigación se considera descriptivo-interpretativo y analítico ex-post-facto y se presentan datos obtenidos en los resultados mediante la aplicación de cuestionarios.

Universo y técnica de muestreo.

Se trabajó con el tipo de muestreo probabilístico estratificado. Según Hernández, Fernández y Baptista (2010) señala: "Muestreo en el que la población se divide en segmentos y se seleccionará una muestra para cada segmento" (p.180). El muestreo probabilístico estratificado servirá para tener datos más exactos de cada estrato y así también para comparar los resultados obtenidos entre uno y otro.

Para conocer la cifra constante de la muestra por estrato, se tendrá que dividir la muestra total (30\% de la población de docentes y $70 \%$ de la población de estudiantes) entre la población total con base en la siguiente fórmula:

$\mathrm{M}=\mathrm{n} / \mathrm{N}$ Donde: $\mathrm{n}=$ Muestra; $\mathrm{N}=$ Población

Remplazando tenemos lo siguiente:

$133=$ Muestra docentes; $906=$ Muestra estudiantes

$445=$ Población docentes; $1209=$ Población estudiantil

Donde $133 / 445=$ a 0.3 (cifra constante a multiplicar en cada estrato docente).

Donde $906 / 1209$ = a 0.7 (cifra constante a multiplicar en cada estrato estudiantil).

Para obtener el tamaño de la muestra en cada estrato, se procedió a dividir la población por cada tipo de grupo entre la fracción constante que, para nuestro caso, es 0.3 en docentes y 0.7 en estudiantes y así obtendremos el resultado en cada segmento de la población.

A continuación, la cantidad de usuarios que se tiene en cada segmento de las dos universidades en Ecuador.

Muestreo de las Universidades.

El proceso de muestreo en las dos universidades de estudio se lo realizó en grupos de docentes y de estudiantes de cada universidad como se observa en la siguiente Figura 1: 
FIGURA 1

Figura 1: Datos de la muestra de docentes y estudiantes de las dos universidades

\begin{tabular}{lllll} 
Tipo de Usuario & Población & Constante & Resultado & Muestra \\
\hline Docentes ULVR & 351 & 0.3 & 105.3 & 105 \\
\hline $\begin{array}{l}\text { Docentes } \\
\text { UISRAEL }\end{array}$ & 94 & 0.3 & 28.2 & 28 \\
\hline $\begin{array}{l}\text { TOTAL Docentes } \\
\text { Estudiantes ULVR }\end{array}$ & $\mathbf{4 4 5}$ & $\mathbf{0 . 3}$ & $\mathbf{1 3 3 . 5}$ & $\mathbf{1 3 3}$ \\
\hline $\begin{array}{l}\text { Estudiante } \\
\text { UISRAEL }\end{array}$ & $\mathbf{4 0 2}$ & 0.75 & 605.25 & 605 \\
\hline $\begin{array}{l}\text { TOTAL } \\
\text { Estudiantes }\end{array}$ & $\mathbf{1 2 0 9}$ & $\mathbf{0 . 7 5}$ & 301.5 & 301 \\
\hline $\begin{array}{l}\text { TOTAL } \\
\text { GENERAL }\end{array}$ & $\mathbf{1 6 5 4}$ & & $\mathbf{9 0 6 . 7 5}$ & $\mathbf{9 0 6}$ \\
\hline
\end{tabular}

Fuente: Elaboración propia

Para la selección de los usuarios a ser encuestados dentro de cada estrato, se utilizará el muestreo probabilístico sistemático; Hernández, Fernández y Baptista (2010) que menciona: "este proceso de selección es muy útil e implica elegir dentro de una población $\mathrm{N}$ un número de $\mathrm{n}$ elementos a partir de un intervalo K” (p. 184).

Hernández, Fernández y Baptista (2010) complementa diciendo: “Este último (k) es un intervalo que va a ser determinado por el tamaño de la población y el tamaño de la muestra” (p. 184). Entonces para la selección de cada usuario a ser encuestado se tuvo la siguiente fórmula:

$\mathrm{K}=\mathrm{N} / \mathrm{n}$; Donde: $\mathrm{n}=$ Muestra; $\mathrm{N}=$ Población

Remplazando tenemos lo siguiente:

$133=$ Muestra docentes; $906=$ Muestra estudiantes

$445=$ Población docentes; $1209=$ Población estudiantil

Donde 133 / 445 = a 0.3 (cifra constante a multiplicar en cada estrato docente).

Donde $906 / 1209$ = a 0.7 (cifra constante a multiplicar en cada estrato estudiantil).

Se infiere con el resultado que se entregó en la encuesta a 1 de cada 3 docentes y 3 de cada 4 estudiantes pertenecientes a las Universidades en estudio.

Instrumento de recolección de la información.

Para la recolección de la información se utilizó el instrumento del cuestionario.

Cuestionario. El cuestionario en la presente investigación se basó en el trabajo de Lareki, Martínez de Morentin, y Amenabar (2010) y se complementaron con los aportes de Georgina y Hosford (2009) que establecieron una serie de clasificaciones sobre el uso de TIC en la formación universitaria. Se tomó como modelo el trabajo de Lareki, Martínez y Amenabar (2010) publicado en la revista Computer and Education denominado Towards and Efficient Training of University Faculty on Icts,; tratando de recopilar la información que más se ajuste a la realidad, se tomó en cuenta a Schwarz y Wimmers (1999) las recomendaciones de Holt, Bury, Bersten, Skowronski y Vedig (1992) en el diseño de las herramientas para la 
recopilación de datos, así como las sugerencias dadas por Norman, Friedman, Norman y Stevenson (2001) en la creación de una encuesta en línea.

Las preguntas del cuestionario se agruparon de la siguiente forma: a) uso intensivo de TIC; b) herramientas para la educación virtual; c) uso de herramientas de Internet; d) herramientas tecnológicas; e) herramientas para la búsqueda y uso de la información (ver anexo).

Para tener indicadores más consistentes en el cuestionario, se realizó un primer borrador de la encuesta y se hizo partícipe a las autoridades de las universidades para que validen las preguntas que se proponían y se obtiene como resultado un cuestionario que fue evaluado por docentes de las dos universidades y especialistas en las respectivas áreas del conocimiento. Después de varias reuniones de trabajo, se definieron las preguntas finales del cuestionario cuyo objetivo es informarnos sobre el real conocimiento que tienen los estudiantes y docentes en cuanto a TIC se refiere en el proceso de enseñanza - aprendizaje.

Posteriormente, el cuestionario fue validado por dos grupos de expertos. El primer grupo se consideró a directores administrativos de la Universidad Nacional Mayor de San Marcos (UNMSM), de Lima - Perú. En el segundo grupo se consideró a especialistas en la aplicación de las TIC en la educación, de la UNMSM. Después, un estudio piloto se llevó a cabo la aplicación con el fin de probar el cuestionario y poder recoger las opiniones de los docentes y estudiantes sobre su validez.

El cuestionario final fue digitalizado en una plataforma en línea y puesta a disposición para la aplicación de este, el cual fue realizado hasta el mes de julio del 2016; cabe resaltar que en el mismo sistema se almacenaron todos los datos respondidos por estudiantes y docentes, y se agregó la gran participación y ayuda de directores, administrativos, decanos, docentes y autoridades en toda la logística.

Una encuesta anterior fue aplicada a estudiantes de las universidades antes mencionadas y posteriormente se decidió realizar la encuesta dirigida a los docentes con la finalidad de poder analizar la brecha que existía entre estos dos grupos de estudio.

Procedimientos y análisis de datos.

Una vez realizado el proceso de validación del cuestionario, tal como se describe en párrafos anteriores, se precedió a la aplicación de manera dual, tanto en línea como presencial para los dos grupos de estudio.

En los estudiantes, para la aplicación de forma virtual, se tuvo colaboración de parte de las autoridades para poder obtener la autorización de ocupar sus laboratorios y hacer que los estudiantes antes de iniciar sus clases normales, completen el cuestionario. Para la aplicación de forma presencial, se ingresó a las aulas y con la colaboración de los docentes se procedió a la aplicación del cuestionario.

Para los docentes se tuvo mayor facilidad, todos lo aplicaron de la forma virtual, para la primera institución la UISRAEL se lo realizó en una de sus capacitaciones; se les pidió que llenaran la encuesta virtual, muchos la completaron desde sus teléfonos; para la otra institución ULVR se pidió a las autoridades convocar a los docentes a los laboratorios y ahí se les realizó la aplicación del cuestionario.

Una vez aplicado el cuestionario a los a 133 docentes y 906 estudiantes, se subió la información de los 85 estudiantes que lo realizaron de manera presencial al sistema de encuestas en línea que se utilizó, debido a que en el propio sistema muestra los resultados de los datos del cuestionario aplicado. El análisis de estos datos se ejecutó mediante la aplicación de criterios de comparación entre los resultados de docentes y los resultados de estudiantes, siempre que las comparaciones sean de la misma categoría.

\section{Resultados}

\section{Edad, género y tipo de los contratos.}

Para la presente investigación se tomó como base Ley de Educación Superior del Ecuador del 12 de octubre del 2010, que estipula los tipos de contratos para los docentes son tres clases: tiempo completo, medio tiempo y tiempo parcial, pero para efectos de mejorar las evaluaciones, es necesario tener docentes contratados a tiempo completo. Existe también la reglamentación de la contratación docente donde indica que el proceso 
de ingreso a las Universidades es mediante un concurso de méritos, oposición y clases demostrativas ante una comisión especializada integrada por miembros de la universidad como paso previo para la contratación. De la misma manera la ley mencionada estipula un examen de admisión obligatoria para los estudiantes que deseen acceder a las IES públicas o privadas.

Bajo esta premisa, se dividió en grupo de edades y género tanto a docentes como estudiantes; además a los docentes en tipos de contratos y a los estudiantes por nivel de estudios. Cabe resaltar que los datos de género en docentes no fueron relevantes, por eso no se tomaron en cuenta, sin embargo en los estudiantes son mayormente mujeres las que están en las aulas universitarias, con un 59\% del total.

En cuanto al nivel de estudios de los estudiantes la gran mayoría se concentra en los primeros niveles que corresponden a los estudiantes de menores edades y según avanzan las edades, la cantidad de estudiantes disminuye, así como cambia la cantidad mayoritaria de estudiantes por género, siendo varones los que más estudian en los niveles más altos y de edades más avanzadas.

En cuanto a los docentes la mayor parte se agrupan entre los $40-44$ años, representan el $20 \%$ de toda la muestra, y también podemos ver que son en un $61 \%$ docentes con contratos a tiempo completo, tan solo el $31 \%$ a medio tiempo y apenas el $8 \%$ de docentes a tiempo parcial.

Para analizar los datos de una manera adecuada, no se han tomado en cuenta las cifras de extremos, debido a que pueden distorsionar una tendencia porque son muy pocas; así; de los grupos de edades de los docentes universitarios, los menores a 25 años y los mayores de 60 años, que tan solo es un docente del primer grupo y 7 del segundo grupo y para los estudiantes no se tomó en cuenta los datos de mayores de 50 años, pues son solo dos estudiantes y no representan ninguna tendencia. Sin embargo, para los resultados generales se tomó en cuenta todos los datos obtenidos.

\section{Herramientas: equipos electrónicos de computación.}

Se realizó un análisis de varios tipos de herramientas tecnológicas que utilizan tanto docentes como estudiantes para realizar actividades dentro del proceso de enseñanza aprendizaje, que no solo está dirigida a las clases en aula, sino representa también en la preparación de clase, resolución de tareas y otras actividades. Estos datos de recursos utilizados están agrupados por edades para docentes y estudiantes además del tipo de contrato para docentes, de manera que se ha obteniendo las siguientes Tablas 1 y 2 resultantes:

TABLAS 1 Y 2

Tablas 1 y 2: Uso de equipos electrónicos y de computación

Tabla 1 (Docentes) $\quad$ Tabla 2 (Estudiantes)

\begin{tabular}{lrrrrrrrrrrrr} 
Edad & Proy & PC & Laptop & Tablet & $\begin{array}{r}\text { Smart } \\
\text { Phone }\end{array}$ & Edad & Proy & PC & Laptop & Tablet & $\begin{array}{r}\text { Smart } \\
\text { Phone }\end{array}$ & $\begin{array}{l}\text { Pizarra } \\
\text { Digital }\end{array}$ \\
\hline $25-29$ & 63,3 & 70,0 & 75,6 & 62,2 & 58,9 & -20 & 35,9 & 71,2 & 55,4 & 45,5 & 71,2 & 1,1 \\
\hline $30-34$ & 58,0 & 57,6 & 82,6 & 19,3 & 82,6 & $20-24$ & 46,4 & 75,2 & 58,6 & 37,8 & 74,2 & 2,3 \\
\hline $35-39$ & 66,7 & 80,0 & 86,7 & 60,0 & 43,3 & $25-29$ & 45,7 & 76,9 & 65,9 & 38,2 & 61,3 & 2,9 \\
\hline $40-44$ & 68,8 & 87,5 & 87,5 & 27,1 & 81,3 & $30-34$ & 36,0 & 74,0 & 86,0 & 52,0 & 70,0 & 6,0 \\
\hline $45-49$ & 77,9 & 92,3 & 57,2 & 45,4 & 57,2 & $35-39$ & 45,5 & 81,8 & 81,8 & 54,6 & 63,6 & 0,0 \\
\hline $50-54$ & 70,6 & 90,5 & 78,4 & 37,2 & 59,7 & $40-44$ & 62,5 & 87,5 & 62,5 & 37,5 & 62,5 & 0,0 \\
\hline $55-59$ & 85,7 & 92,9 & 53,6 & 46,4 & 75,0 & $45-49$ & 50,0 & 66,7 & 83,3 & 50,0 & 33,3 & 16,7 \\
\hline+60 & 77,8 & 88,9 & 66,7 & 22,2 & 66,7 & $50-54$ & 0,0 & 0,0 & 100,0 & 0,0 & 100,0 & 0,0 \\
\hline Prom & 71,1 & 82,5 & 73,5 & 40,0 & 65,6 & Prom & 46,0 & 76,0 & 71,0 & 45,0 & 62,0 & 4,0 \\
\hline
\end{tabular}

Fuente: Elaboración propia 
En la comparación de la Tabla 1 y la Tabla 2 se pudo observar lo siguiente:

No se encontraron mayores diferencias en el uso de las herramientas tecnológicas, por ejemplo el uso de la PC sigue predominando en los dos grupos con la diferencia de que el $82,5 \%$ de los docentes lo utiliza y en los estudiantes es en menor porcentaje en un $74 \%$. Debido probablemente a que los estudiantes cada vez utilizan más los teléfonos inteligentes que las PC, así lo demuestra la comparación de las Tablas donde los estudiantes usan para sus actividades escolares un 70\% los teléfonos inteligentes frente a un 65,6\% de los docentes.

Un dato que cabe resaltar es que los docentes emplean el retroproyector para sus clases en un $71 \%$ y los estudiantes tan solo un $42 \%$, esto probablemente porque los estudiantes solo lo hacen en días específicos y no lo perciben como una herramienta útil ni de uso diario.

TABLA 3 Y 4

Tablas 3 y 4 . Uso sistemas de educación virtual

Tabla 3 (Docentes) $\quad$ y Tabla 4 (Estudiantes)

\begin{tabular}{|c|c|c|c|c|c|c|c|c|c|}
\hline $\begin{array}{l}\text { Rang } \\
0\end{array}$ & $\begin{array}{l}\text { Moodl } \\
\mathrm{e}\end{array}$ & $\begin{array}{l}\text { Educati } \\
\text { va }\end{array}$ & $\begin{array}{l}\text { Blackboa } \\
\text { rd }\end{array}$ & $\begin{array}{l}\text { Ningun } \\
\text { a }\end{array}$ & $\begin{array}{l}\text { Rang } \\
0\end{array}$ & $\begin{array}{l}\text { Moodl } \\
\text { e }\end{array}$ & $\begin{array}{l}\text { Educati } \\
\text { va }\end{array}$ & $\begin{array}{l}\text { blackboa } \\
\text { rd }\end{array}$ & $\begin{array}{l}\text { Ningun } \\
\text { a }\end{array}$ \\
\hline $25-29$ & 0,0 & 51,1 & 13,3 & 28,9 & -20 & 9,3 & 39,3 & 1,4 & 25,4 \\
\hline $30-34$ & 16,3 & 23,5 & 3,0 & 26,9 & $20-24$ & 11,6 & 30,1 & 3,3 & 22,2 \\
\hline $35-39$ & 36,7 & 23,3 & 3,3 & 43,3 & $25-29$ & 13,3 & 31,2 & 1,7 & 21,4 \\
\hline $40-44$ & 10,4 & 31,9 & 0,0 & 29,9 & $30-34$ & 20,0 & 34,0 & 2,0 & 14,0 \\
\hline $45-49$ & 12,8 & 56,9 & 9,2 & 7,7 & $35-39$ & 18,2 & 27,3 & 0,0 & 27,3 \\
\hline $50-54$ & 44,4 & 16,9 & 4,8 & 12,6 & $40-44$ & 12,5 & 25,0 & 0,0 & 62,5 \\
\hline $55-59$ & 0,0 & 19,6 & 0,0 & 53,6 & $45-49$ & 16,7 & 66,7 & 0,0 & 16,7 \\
\hline $60+$ & 33,3 & 22,2 & 11,1 & 33,3 & & & & & \\
\hline Prom & 19,2 & 30,7 & 5,6 & 29,5 & Prom & 14,5 & 36,2 & 1,2 & 27,1 \\
\hline
\end{tabular}

Fuente: Elaboración propia

En el análisis de las Tablas 3 y 4 se puede observar que existen muy pocos estudiantes y docentes que utilizan los sistemas de educación virtual, siendo una de las principales herramientas en la educación actual, tan solo en promedio el $30,7 \%$ de los docentes y el $37 \%$ de los estudiantes en todos los grupos de edades usa el sistema E-ducativa, seguido por la plataforma Moodle con un 19,2\% y 13\%, respectivamente.

Estos sistemas que son de mayor uso e impacto en los grupos de estudio es probablemente debido a que son los sistemas instalados en las universidades de estudio; E-ducativa en la ULVR y Moodle en UISRAEL, siendo la ULVR la que tiene mayor cantidad de estudiantes y docentes. Además de ser un parámetro de medición en la calidad educativa en las IES del Ecuador, se debería fomentar un mayor uso de estas.

Cabe resaltar que los estudiantes de 45 a 49 años de edad son los que más utilizan la plataforma Educativa con un 66,67\% siendo este el porcentaje más alto entre estudiantes y docentes de todos los grupos de edad. En relación con la plataforma de Moodle, los grupos de mayor uso son los que están en las edades comprendidas entre 50-54 años, en los docentes con un $44,4 \%$ y de 30 - 34 años en los estudiantes con un $20 \%$. 
TABLA 5 Y 6

Tablas 5 y 6 . Uso de Recursos tecnológicos online de investigación.

Tabla 5 (Docentes)

\begin{tabular}{|c|c|c|c|}
\hline Rango & $\begin{array}{l}\text { Bibliotecas } \\
\text { Digitales }\end{array}$ & $\begin{array}{l}\text { Catálogos } \\
\text { Digitales }\end{array}$ & $\begin{array}{l}\text { Repositol } \\
\text { Digitales }\end{array}$ \\
\hline $25-29$ & 93,3 & 42,2 & \\
\hline $30-34$ & 84,5 & 28,8 & \\
\hline $35-39$ & 93,3 & 30,0 & \\
\hline $40-44$ & 84,7 & 31,9 & \\
\hline $45-49$ & 88,2 & 66,2 & \\
\hline $50-54$ & 84,4 & 70,6 & \\
\hline $55-59$ & 73,2 & 32,1 & \\
\hline $60+$ & 77,8 & 11,1 & \\
\hline Prom & 84,9 & 39,1 & \\
\hline $\begin{array}{l}\text { Bibliotecas } \\
\text { Digitales }\end{array}$ & $\begin{array}{l}\text { Catálogos } \\
\text { Digitales }\end{array}$ & $\begin{array}{l}\text { Reposi } \\
\text { Digitale }\end{array}$ & \\
\hline
\end{tabular}

Tabla 6 (Estudiantes)

\begin{tabular}{rrr}
\hline 73,7 & 32,5 & 9,9 \\
\hline 73,2 & 33,8 & 15,2 \\
\hline 76,9 & 35,8 & 16,2 \\
\hline 82,0 & 56,0 & 18,0 \\
\hline 100,0 & 72,7 & 18,2 \\
\hline 62,5 & 12,5 & 37,5 \\
\hline 83,3 & 50,0 & 16,7 \\
\hline & & \\
\hline 78,8 & 41,9 & 18,8 \\
\hline
\end{tabular}

Fuente: Elaboración propia

En el análisis general se puede ver que los estudiantes tienen un promedio de uso menor que el de los docentes en el empleo de estas herramientas de investigación aunque los porcentajes son relativamente altos de $84,9 \%$ para las bibliotecas digitales en docentes y 78,80\% en los estudiantes, cabe resaltar que esto responde a que las bibliotecas digitales que operan en las universidades son parte de una exigencia de los organismos de control de la Educación Superior en el Ecuador y estos hacen un seguimiento anual de uso de las bibliotecas digitales por parte de los docentes y estudiantes.

Los porcentajes de uso más llamativos en los grupos de edades es para los docentes que están entre 25-29 años con un $93 \%$ de uso aproximadamente y los estudiantes de 35 y 39 años usa el $100 \%$.

Utilización de software en Internet. En cuanto a la utilización del software en el Internet, se analizaron seis herramientas básicas de utilización para el proceso de enseñanza aprendizaje y se obtuvieron los siguientes datos: 
TABLA 7 Y 8

Tablas 7 y 8: uso de software en Internet

Tabla 7. (Docentes).

\begin{tabular}{lrrrrrr} 
Rango & Blog & Browser & Correo & $\begin{array}{l}\text { Redes } \\
\text { Sociales }\end{array}$ & \multicolumn{1}{l}{ Confer } & Apps \\
\hline $25-29$ & 0,0 & 68,9 & 71,1 & 33,3 & 45,6 & 11,1 \\
\hline $30-34$ & 11,4 & 80,7 & 76,5 & 22,4 & 13,3 & 54,9 \\
\hline $35-39$ & 16,7 & 83,3 & 90,0 & 22,0 & 33,3 & 16,7 \\
\hline $40-44$ & 15,3 & 43,8 & 64,6 & 17,1 & 16,7 & 25,7 \\
\hline $45-49$ & 42,6 & 24,6 & 79,0 & 30,2 & 47,7 & 38,5 \\
\hline $50-54$ & 38,3 & 65,8 & 60,0 & 30,4 & 44,8 & 13,9 \\
\hline $55-59$ & 39,3 & 78,6 & 73,2 & 22,1 & 39,3 & 32,1 \\
\hline $60+$ & 11,1 & 55,6 & 77,8 & 33,3 & 0,0 & 11,1 \\
\hline Prom & 21,8 & 62,6 & 74,0 & 26,4 & 30,1 & 25,5 \\
\hline
\end{tabular}

$y$

Tabla 8. (Estudiantes).

\section{Fuente: Elaboración propia}

En cuanto a los programas en Internet que se utilizan para el desarrollo del proceso enseñanza aprendizaje, (Tablas 7 y 8); se pudo observar varios aspectos entre los principales, los estudiantes utilizan en mayor cantidad los blogs, el navegador de Internet y el correo electrónico, sin ser muy grande la diferencia, pero en promedio el uso de las redes sociales, la videoconferencia y las aplicaciones tecnológicas, en promedio quienes mayormente utilizan son los docentes. Esto básicamente se puede explicar porque estos sistemas los usa el alumnado más para el ocio y no para la educación, al contrario de los docentes que su mayor utilidad lo dan para el proceso enseñanza aprendizaje.

Los datos relevantes son que los docentes usan el correo electrónico en un promedio el $74 \%$, mientras que los estudiantes lo utilizan en un $81,47 \%$. Los estudiantes que más emplean el correo y están sobre el promedio son los estudiantes de 30 a 44 años, llegando a 100\% ene estudiantes de 30 a 35 años, con los estudiantes más jóvenes que tienen los promedios más bajos.

Cabe resaltar que los blogs, videoconferencias y aplicaciones tecnológicas específicas para la educación tiene promedios muy bajos de uso, por lo que llama la atención que siendo herramientas que permiten o facilitan el proceso de enseñanza aprendizaje, se utilicen con poca frecuencia. En cuanto a las redes sociales y en especial el Facebook, los docentes para sus actividades académicas solo utilizan un $30 \%$ y $35 \%$ en el caso de los estudiantes, que son porcentajes relativamente bajos si consideramos que es una herramienta de gran ayuda para fines académicos y en la actualidad lo usan en su gran mayoría para el ocio y descansos en sí.

Utilización de programas de escritorio. En cuanto a la utilización del software de escritorio, que se refieren a los programas que están instalados en una computadora, en el Internet, se preguntó sobre cinco herramientas muy utilizadas en los procesos de enseñanza aprendizaje y se obtuvieron los siguientes datos: 
TABLA 9 Y 10

Tablas 9 y 10: Uso de programas en Escritorio

Tabla 9. (Docentes).

Tabla 10. (Estudiantes).

\begin{tabular}{|c|c|c|c|c|c|c|c|c|c|c|}
\hline \multirow[b]{2}{*}{ Rango } & \multirow[b]{2}{*}{ Word } & \multicolumn{2}{|l|}{ Power } & \multicolumn{2}{|r|}{ Proq Diseño } & \multirow[b]{3}{*}{ Word } & \multirow[b]{2}{*}{ Power } & \multirow[b]{3}{*}{ Excel } & \multirow{3}{*}{\multicolumn{2}{|c|}{$\begin{array}{l}\text { Prog } \\
\text { Diseño } \\
\text { WEB }\end{array}$}} \\
\hline & & Point & Excel & SPSS & WEB & & & & & \\
\hline $25-29$ & 86,7 & 71,1 & 75,6 & 11,1 & 17,8 & & Point & & & \\
\hline $30-34$ & 91,7 & 88,6 & 85,6 & 4,2 & 25,8 & 89,0 & 84,5 & 70,3 & 3,7 & 9,0 \\
\hline $35-39$ & 93,3 & 86,7 & 83,3 & 10,0 & $\overline{13,3}$ & 89,4 & 82,1 & 80,8 & 4,3 & 10,6 \\
\hline $40-44$ & 91,7 & 82,6 & 71,5 & 2,1 & 2,1 & 88,4 & 78,6 & 82,7 & 4,6 & 11,0 \\
\hline $45-49$ & 97,4 & 85,6 & 74,9 & 2,6 & 2,6 & 94,0 & 84,0 & 90,0 & 8,0 & 12,0 \\
\hline $50-54$ & 97,0 & 84,4 & 74,9 & 19,7 & 15,6 & 100,0 & 100,0 & 90,9 & 9,1 & 27,3 \\
\hline $55-59$ & 92,9 & 92,9 & 73,2 & 25,0 & 7,1 & 100,0 & 87,5 & 87,5 & 0,0 & 12,5 \\
\hline $60+$ & 88,9 & 77,8 & 66,7 & 0,0 & 11,1 & 100,0 & 100,0 & 100,0 & 0,0 & 0,0 \\
\hline Prom & 92,4 & 83,7 & 75,7 & 9,3 & 11,9 & & & & & \\
\hline & & & & & & 94,4 & 88,1 & 86,0 & 5,9 & 11,8 \\
\hline
\end{tabular}

Fluente: Elaboración propia

En los programas de escritorio se pudo destacar varios aspectos en el estudio, entre los principales, los estudiantes utilizan en mayor cantidad el software que los docentes, lo que no ocurría en las herramientas o dispositivos tecnológicos como se describió en las Tablas ( 1 y 2); también concuerda con los datos que mostraron que los estudiantes utilizan en porcentajes altos, similares a los docentes la laptop, pero el teléfono inteligente, que es el medio por el cual se conectan a todos los programas o software de estudio es donde su uso daba una mayor brecha.

Los datos en cuanto al Word y PowerPoint, los docentes los utilizan un $92,4 \%$ y $83,7 \%$ respectivamente, mientras que los estudiantes en un $94,40 \%$ y $88,1 \%$ respectivamente; sin dejar de lado el Excel con $86 \%$ para los estudiantes y $75 \%$ para los docentes; es decir las herramientas más utilizadas siguen siendo las de Microsoft a la hora de realizar actividades del proceso de enseñanza aprendizaje, pero cada vez con mayor fuerza los estudiantes están utilizando también las herramientas en Internet.

\section{Discusión}

Los resultados demuestran que es imprescindible elaborar políticas para el uso de TIC en el proceso de enseñanza aprendizaje y tomar en cuenta las múltiples y diversas herramientas que facilitan este proceso.

El estudio refleja que existe una brecha entre docentes y estudiantes; un posible estudio complementario debería analizar el planeamiento estratégico de cada universidad para conocer si las TIC se han fortalecido con una mirada de mediano y largo plazo.

Los nuevos movimientos como el Open Data obligan a las universidades en el fortalecimiento en el uso de TIC. El Open Data son datos generados por diversas instituciones, que brinda la posibilidad de ser utilizados, reutilizados y redistribuidos libremente por cualquier persona, pueden compartirse y enriquecer el conocimiento, es un insumo fundamental para las organizaciones académicas porque permiten la generación de investigación de forma sostenida; estos datos permiten el análisis crítico y apoyan el desarrollo de las sociedades y obviamente no funcionarían sin las TIC y la programación. 
La brecha que existe en el uso de la tecnología básica de la información y comunicación en el proceso de enseñanza aprendizaje cada vez se va reduciendo entre los docentes y estudiantes que son los principales gestores de dicho proceso, esto básicamente por dos componentes; el primero es que el rol de los docentes en la actualidad, en todas las instituciones educativas superiores del mundo, han tenido una constante capacitación y adiestramiento en el manejo de las herramientas tecnológicas básicas y no solo dentro de las universidades también en todo el entornos social, son herramientas indispensables para el desarrollo cotidiano, esto hace que los docentes estén cada día más preparados para asumir nuevos retos y nuevas tecnologías, en el desarrollo de sus clases; se pudo comprobar que en las instituciones de estudio se realizan capacitaciones en las plataformas tecnológicas cada inicio de semestre.

El segundo componente es que la tecnología en sí se va desarrollando cada día de mejor manera con la finalidad de poder estar al servicio de los docentes y estudiantes con mayores facilidades; el éxito que pueden tener estas herramientas con los docentes básicamente está dado por el real aporte de entregar en cuanto al ahorro de tiempo que experimentan los docentes en: preparar sus clases, corrección de tareas, evaluaciones y exámenes.

Los estudiantes están en constante evolución con respecto al uso de las tecnologías de información por lo cual la adopción de nuevas tecnologías en el proceso de enseñanza aprendizaje se les facilita abiertamente. Sin embargo, es importante considerar que los estudiantes cada vez utilizan con menos frecuencia las tecnologías de comunicación básica que van quedando en el pasado como computadoras, proyectores, computadoras portátiles y demás dispositivos electrónicos así como los respectivos programas; por el mismo hecho de que están en la vanguardia y pierden el interés por esta tecnología pasada; por lo que los docentes en busca de mejorar el rendimiento del proceso de enseñanza aprendizaje, también debe estar alineado siempre a la tecnología vanguardista.

Para reducir la brecha en la tecnología básica de información y comunicación en las universidades estudiadas, a los docentes se les dota de capacitación constante, que les permite estar al día en el uso de tecnologías de información relacionadas con el proceso de enseñanza aprendizaje, lo que mejora la interactividad con los estudiantes y el proceso de generación de conocimiento.

La brecha en el uso de la tecnología fue reduciéndose considerablemente desde que los docentes entendieron que su uso no solo es beneficioso para los estudiantes o porque se necesita una acreditación, sino también porque a ellos les trajo muchos beneficios una tecnología bien implementada. Debido a que redujo considerablemente los tiempos en preparación de clase, en corregir exámenes y pruebas, en tomar lecciones, en enviar y explicar los trabajos que debían realizar, es decir, el tiempo en tareas operativas que les llevaba más del 60\% de su tiempo, ahora lo pueden aprovechar en otras actividades que encaminen al mejoramiento de la investigación, de la generación del conocimiento y por su puesto al proceso de enseñanza aprendizaje.

Mientras más se reduce la brecha tecnológica en el proceso de enseñanza aprendizaje entre los docentes y estudiantes; más el docente puede llegar a impartir sus conocimientos a través de sus cursos o lecciones a mayor cantidad de estudiantes, llegando en la actualidad a tener cursos que desarrollan los docentes para más de 500.000 estudiantes registrados simultáneamente y creando conocimiento con actividades completamente interrelacionadas.

La brecha que existe entre estudiantes y docentes en esta tecnología básica de información, no es muy grande ni muy considerable, básicamente porque los docentes en las universidades de estudio tienen una constante capacitación, obedeciendo a las políticas institucionales que buscan la mejora continua en la calidad académica. También porque las instituciones de control de las universidades en el Estado ecuatoriano tienen políticas de evaluación constante a las IES y varios de los indicadores de evaluación está basado en las competencias de los docentes y las actividades de capacitación que estos presentan en cuanto a manejo y administración de tecnologías básicas de la información en el proceso de enseñanza aprendizaje. Esto ha generado una cultura de evaluación y mejora continua en todos los miembros de la comunidad Universitaria. 
Cabe aclarar que ya para el tipo de tecnología de comunicación de vanguardia, estas son las basadas en Internet y que principalmente permiten el desarrollo de la generación del conocimiento de manera eficiente, se pudo observar una brecha mucho mayor entre docentes y estudiantes, que en el uso de la tecnología básica de comunicación e información.

Se pudo observar que las tecnologías de comunicación e información de vanguardia son poco utilizadas para los fines académicos en las universidades estudiadas, pues en su gran mayoría se las usa para ocio. Esto convierte a la comunidad universitaria de estudiantes en consumidores de tecnología y no en lo que deberían ser, generadores de conocimiento a través de la tecnología. Se puede observar que están listos para pasar al siguiente nivel de aprendizaje, el de colaboración con todo el mundo, porque utilizan las mejores aplicaciones con aparatos electrónicos de última generación, es decir, tienen todo listo para dar el gran salto. Sin embargo, es posible constatar que los docentes aún no tienen ese conocimiento en este tipo de aplicaciones, pero sí los equipos electrónicos para acceder a todo este nuevo mundo de la generación del conocimiento colaborativo a través de las nuevas TIC.

No se puede terminar esta discusión de resultados, sin resaltar que el presente estudio se hizo en tan solo dos universidades del Ecuador, una para Quito y otra para Guayaquil; por lo cual las conclusiones podrían ser muy segmentadas, además no se han tomado en cuenta las universidades públicas que son mayoría y es donde se encuentra la mayor cantidad de estudiantes y docentes, por lo tanto se propone realizar más estudios a profundidad para complementar el presente trabajo, y así obtener conclusiones cada vez más contundentes.

\section{Conclusiones}

- Es imprescindible para las universidades establecer políticas para el uso de TIC en el proceso de enseñanza aprendizaje orientada a la disminución de la brecha digital.

- La mayor parte de los docentes encuestados se agrupan entre los 40-44 años, representan el 20\% de toda la muestra que puede ser considerada una edad media para quien ejerce la docencia y también hay un $61 \%$ de docentes con contratos a tiempo completo, esto significa que hay un grupo mayoritario dedicado exclusivamente a la docencia.

- Las TIC y las herramientas tecnológicas utilizadas por docentes y estudiantes en el proceso de enseñanza aprendizaje se usan para múltiples actividades (clases en aula, preparación de clases, resolución de tareas, por ejemplo). La PC sigue predominando en los dos grupos con la diferencia de que el $82,5 \%$ de los docentes la emplea y en los estudiantes es en menor porcentaje con un $74 \%$ (el $65 \%$ de los estudiantes utiliza teléfonos inteligentes).

- Es limitado el uso de sistemas de educación virtual en docentes y estudiantes, solo en promedio el 30,7\% de los docentes y el 37\% de los estudiantes en todos los grupos de edades utiliza el sistema Educativa, seguido por la plataforma Moodle con un 19,2\% y $13 \%$ respectivamente. Las herramientas de investigación tienen porcentajes de uso de $84,9 \%$ para las bibliotecas digitales en docentes y $78,80 \%$ en los estudiantes

- En el uso de herramientas de Internet (blog, browser, correo electrónico, entre otros) se obtuvieron datos relevantes; mientras los docentes utilizan el correo electrónico en un promedio el $74 \%$ los estudiantes lo utilizan en un $81,47 \%$. Un dato para resaltar es que los estudiantes que más utilizan el correo y están sobre el promedio son los estudiantes de 30 a 44 años, llegando a 100\%. Las otras aplicaciones tecnológicas tienen tasas bajas de utilización.

- El Word y PowerPoint son empleados por los docentes en un $92,4 \%$ y $83,7 \%$ respectivamente, mientras que los estudiantes en un 94,40 y $88,1 \%$ respectivamente; sin dejar de lado el Excel con $86 \%$ para los estudiantes y $75 \%$ para los docentes; estas herrami9entas de Microsoft siguen teniendo predominio en las universidades. 


\section{Referencias BIBLIOgráficAs}

Ávila-Fajardo, G. P., y Erazo, S. C. R. (2011). Propuesta para la medición del impacto de las TIC en la enseñanza universitaria. Educación y educadores, 14(1), 169-188.

Cabero, J. (2014). Formación del profesorado universitario en TIC. Aplicación del método Delphi para la selección de los contenidos formativos.Educación XXI,17(1), 111-132.

Cabero, J. (2015). Reflexiones educativas sobre las tecnologías de la información y la comunicación (TIC). Tecnología, Ciencia y Educación, 1, 19-27.

Esteve, F. (2016). Bolonia y las TIC: de la docencia 1.0 al aprendizaje 2.0. La cuestión universitaria, (5), 58-67.

Fombona, J., y Pascual, M. (2011). Las tecnologías de la información y la comunicación en la docencia universitaria. Estudio de casos en la Universidad Nacional Autónoma de México (UNAM). Educación XXI, 14(2), 79-110.

Fu, J. S. (2013). ICT in education: A critical literature review and its implications. International Journal of Education and Development using Information and Communication Technology, 9(1), 112-125.

Georgina, D. A., y Hosford, C. C. (2009). Higher education faculty perceptions on technology integration and training. Teaching and Teacher Education, 25(5), 690-696.

Guerra, S., González, N. y García-Ruiz, R. (2010). Utilización de las TIC por el profesorado universitario como recurso didáctico. Comunicar: Revista científica iberoamericana de comunicación y educación, (35), 141-148.

Gutiérrez, P., Yuste Tosina, R., Cubo Delgado, S. y Lucero Fustes, M. (2011). Buenas prácticas en el desarrollo de trabajo colaborativo en materias TIC aplicadas a la educación. Profesorado. Revista de currículum y formación de profesorado, 15(1), 179-194.

Hernández, R., Fernández, C., y Baptista, P. (2010). Metodología de la investigación. México: Editorial Mc Graw Hill.

Holt, A. W., Bury, L. K., Bersten, A. D., Skowronski, G. A. y Vedig, A. E. (1992). Prospective evaluation of residents and nurses as severity score data collectors. Critical care medicine, 20(12), 1688-1691.

Johnson, L., Becker, S., Estrada, V. y Freeman, A. (2015). NMC Horizon Report: Edición Educación Superior 2015. Austin, Texas: The New Media Consortium.

Kalogiannakis, M. (2010). Training with ICT for ICT from the trainee's perspective. A local ICT teacher training experience. Education and Information Technologies, 15(1), 3-17. doi: DOI 10.1007/s10639-008-9079-3

Kulakli, A., y Mahony, S. (2014). Knowledge creation and sharing with Web 2.0 tools for teaching and learning roles in so-called University 2.0. Procedia-Social and Behavioral Sciences, 150, 648-657.

Lareki, A., Martínez de Morentin, J. I. y Amenabar, N. (2010). Towards an efficient training of university faculty on ICTs. Computers y Education, 54(2), 491-497. doi: 10.1016/j.compedu.2009.08.032

Marín, V., Vásquez, A. I., Llorente Cejudo, M. C. y Cabero, J. (2012). La alfabetización digital del docente universitario en el Espacio Europeo de Educación Superior. Edutec. Revista Electrónica de Tecnología Educativa, (39), 194.

Martínez, F. y Acosta, Y. (2011). Integración de las tecnologías de la información y la comunicación (TIC) en la universidad: diez propuestas de aprendizaje. Revista Mediterránea de Comunicación, 2(1), 43-58. Recuperado de https://goo.gl/Gp7Tsa

Morales, M., Trujillo Torres, J. M. y Raso Sánchez, F. (2015). Percepciones acerca de la integración de las TIC en el proceso de enseñanza-aprendizaje de la universidad. Pixel-Bit. Revista de Medios y Educación, 46, 103-117.

Nordkvelle, Y. y Olson, J. (2005). Visions for ICT, ethics and the practice of teachers. Education and Information Technologies, 10(1-2), 21-32.

Norman, K. L., Friedman, Z., Norman, K. y Stevenson, R. (2001). Navigational issues in the design of online selfadministered questionnaires. Behaviour y Information Technology, 20(1), 37-45.

Paredes, J. (2013). Políticas educativas públicas sobre TIC en España. Tres décadas donde los docentes universitarios influyeron en el cambio educativo. Revista Fuentes, (13), 45-78.

Rangel, A., y Peñalosa Castro, E. (2013). Alfabetización digital en docentes de educación: construcción y prueba empírica de instrumento de evaluación. Píxel-Bit. Revista de Medios y Educación, 43, 9-23. 
Regueyra, M. G. (2011). Aprendiendo con las TIC: una experiencia universitaria. Revista Electrónica Actualidades Investigativas en Educación, 11, 1-29.

Rodríguez, R. M. (2010). El impacto de las TIC en la transformación de la enseñanza universitaria: repensar los modelos de enseñanza y aprendizaje. Teoría de la Educación. Educación y Cultura en la Sociedad de la Información, 11(1), 32-68.

Rosario, H. J. y Vásquez Melo, L. F. (2012). Formación del docente universitario en el uso de TIC. Caso universidades públicas y privadas. Pixel-Bit. Revista de Medios y Educación, (41), 163-171.

Rozo, A. C. y Prada, M. (2013). Panorama de la formación inicial docente y TIC en la Región Andina. Revista Educación y Pedagogía, 24(62), 191-204.

Salinas, J. (2004). Innovación docente y uso de las TIC en la enseñanza universitaria. International Journal of Educational Technology in Higher Education (ETHE), 1(1).

Schwarz, P. M. y Wimmers, E. L. (1999). Transforming heterogeneous data with database middleware: Beyond integration. Data Engineering, 31.

Sigalés, C. (2004). Formación universitaria y TIC: nuevos usos y nuevos roles. RUSC. Universities and Knowledge Society Journal, 1(1)

UNESCO (2005). Hacia las sociedades del conocimiento. París: Publicaciones Unesco.

Van Der Vyver, G. (2009). The search for the adaptable ICT student. Journal of Information Technology Education: Research, 8(1), 19-28.

Venables, A. y Tan, G. (2012). Measuring up to ICT Teaching and Learning Standards. Issues in Informing Science y Information Technology, 9, 29-40.

Vera, J. Á., Torres, L. E., y Martínez, E. (2014). Evaluación de competencias básicas en TIC en docentes de Educación Superior en México. Píxel-Bit. Revista de Medios y Educación, (44), 143-155.

\section{Apéndice}

\section{Cuestionario de Buenas Prácticas en TIC}

Datos demográficos

- Edad:

- Género: MoF

- Estudiantes (Nivel básico, Nivel Medio y Nivel Avanzado): NB, NM, NA

- Docentes (Tiempo Parcial, Medio Tiempo y Tiempo Completo): TP, MT, TC

CUESTIONARIO: El siguiente cuestionario se refiere a los materiales y recursos TIC'S que utilizan los docentes y estudiantes en las Actividades Generales de la enseñanza- aprendizaje (Recibir y emitir clase, preparación de clase, preparación de tarea, entre otros)

Equipos de computación y comunicación (Uso)

- Proyector

- Computadora

- Tablets

- Teléfonos inteligentes (Smartphone)

- Phablets

- Pizarras digitales

- Memorias USB / pen drives 
Plataformas tecnológicas que use su facultad

- Moodle

- E-ducativa

- Ninguna

- Otros (especifique cuál)

Herramientas de Internet

- Mailing list (listas de interés)

- Blogs

- Navegadores de Internet

- Correo electrónico

- Wikis

- Skype

- Pizarras virtuales

- Hangouts

- Facebook

- Messenger

- Twitter

- Videoconferencia

- Aplicaciones para teléfonos y tablets

- SecondLife

- Otros (Especifique)

Herramientas Tecnológicas

- Procesadores de textos (Word)

- Programas para presentaciones

- Programas de Base de Datos

- Programas de Hojas de cálculo

- Programas de estadísticas SPSS

- Programas de diseño gráfico (Adobe Ilustrator, entre otros)

- Programas de diseño web

- Programas de edición de video

- Creación de páginas PDF

- Programas de reproducción de DVD, blue ray etc.

- Lattes

- Programas de administración de proyectos

- Otros (Especifique)

Recursos de información

- Bases de datos

- Catálogos online

- Repositorio de Datos digitales

- ISI WEB Bases de datos de conocimiento

- Otros (Especifique) 